\title{
Magnetic Properties of Metallic Impurities with Strongly Correlated Electrons
}

\author{
V. JANiŠ AND M. Ringel \\ Institute of Physics, Academy of Sciences of the Czech Republic \\ Na Slovance 2, CZ-182 21 Praha, Czech Republic
}

\begin{abstract}
We study the single impurity Anderson model in an external magnetic field. There are no exact results for the spectral function in this situation. Using a resummation of the diagrammatic expansion we demonstrate that the strong coupling regime in a weak magnetic field is Kondo-like with a quasiparticle resonant peak split into two. We find two exponentially small Kondo scales (temperatures), one for transverse and one for longitudinal spin fluctuations. We show that the salient features of the spectral function in the Kondo regime can be seen already within an extended random phase approximation. To reveal the dependence of the Kondo scales on the bare electron interaction, however, one has to employ a two-particle self-consistency with renormalized vertices. We use the parquet approach to derive the dependence of the Kondo scales on magnetic field.
\end{abstract}

PACS numbers: 71.10.Fd, 71.27.+a

\section{Introduction}

Impurities with correlated electrons in a metallic host has been attracting interest of both theorists and experimentalists for several decades. It is mostly due to the possibility to study formation/screening of local magnetic moments coupled to a sea of conduction electrons. Most of the studies has concentrated on physical properties of the charge and spin symmetric situation. The ground-state of the paragon single-impurity Anderson model (SIAM) is known exactly from the algebraic Bethe ansatz [1]. The most interesting strong-coupling limit is distinguished by the Kondo behavior displaying a number of unique features of one- and two-electron functions. The impurities in metals are physically important in particular for their magnetic properties. Linear-response theory allows one to deduce the reaction to a weak magnetic field already in the spin symmetric solution. Recent realizations of quantum dots attached to magnetically ordered leads, however, demand a knowledge of spectral and, in particular, non-equilibrium properties of charge and/or spin asymmetric situations $[2,3]$.

Unfortunately, there is no exact solution for the spectral function of SIAM in nonzero external magnetic field leading to a spin asymmetry. We have to resort to approximations. There are a few approximate methods either of numerical $[4]$ or analytic $[5,6]$ character, but their results are inconclusive and incomplete. In this paper we reconsider the effects of the applied magnetic field on the strong-coupling regime of SIAM by using a resummation of the diagrammatic expansion for vertex functions. To describe the Kondo regime we extend the approach of Refs. $[7,8]$ to a spin-asymmetric situation. We show that the universal features of the Kondo regime can be recognized already in the random-phase approximation (RPA) for the vertex functions from the electron-hole scattering channels. We introduce a small expansion parameter, being the inverse of the logarithm of the Kondo scale, that controls the diagrammatic expansion beyond RPA. To deduce the dependence of the Kondo scale on the bare interaction strength or the applied magnetic field we have to implement a two-particle self-consistency. We use the one introduced by the parquet approximation with balanced electron-hole and electron-electron multiple scatterings $[7,8]$.

\section{Spin-invariant perturbation theory}

The Hamiltonian of the Anderson impurity embedded in a metallic host with noninteracting conduction electrons reads

$$
\begin{aligned}
\widehat{H} & =\sum_{\boldsymbol{k} \sigma}\left(\epsilon(\boldsymbol{k}) c_{\boldsymbol{k} \sigma}^{\dagger} c_{\boldsymbol{k} \sigma}+V_{\boldsymbol{k}} d_{\sigma}^{\dagger} c_{\boldsymbol{k} \sigma}+V_{\boldsymbol{k}}^{*} c_{\boldsymbol{k} \sigma}^{\dagger} d_{\sigma}\right) \\
& +\sum_{\sigma}\left(E_{d}-\sigma h\right) \widehat{n}_{\sigma}^{d}+U \widehat{n}_{\uparrow}^{d} \widehat{n}_{\downarrow}^{d},
\end{aligned}
$$

where we denoted $\widehat{n}_{\sigma}^{d}=d_{\sigma}^{\dagger} d_{\sigma}$ and $E_{d}$ is the impurity energy level and $h$ is the external magnetic field in units $\mu_{\mathrm{B}}=1$. When calculating thermodynamic properties of the impurity site we can explicitly integrate over the degrees of freedom of the delocalized electrons. To this purpose we standardly replace the local part of the propagator of the mobile electrons by a constant $\Delta(\epsilon)=\pi \sum_{\boldsymbol{k}}\left|V_{\boldsymbol{k}}\right|^{2} \delta(\epsilon-\epsilon(\boldsymbol{k})) \doteq \Delta$ the value of which we set as the energy unit.

The dynamical and spectral functions of SIAM in an external magnetic field are accessible only via approximate schemes. Here we use the many-body diagrammatic representation of impurity Green functions based on the expansion in the interaction strength $U$. We use the 
weak-coupling expansion only for the generation of the diagrammatic contributions to generic functions but do not use the interaction strength as a small expansion parameter. The actual small parameter will be constructed from the Kondo scale.

Standardly, the diagrammatic representation of physical quantities consists of vertices and fermion propagators. We use the Hartree propagators for the fermionic lines and the bare interaction for the vertices. The diagrammatic expansion is then used to determine the self-energy as a dynamical correction to the Hartree static term. There are two important things in our diagrammatic expansion. First, we do not use dynamically renormalized one-electron propagators. We explicitly renormalize only two-particle vertices. Second, to keep the theory globally consistent and to avoid spurious Hartree phase transitions, we employ a static renormalization of the Hartree propagators by means of the renormalized particle and spin densities. We then define the fermion propagators in our expansion to be

$$
G_{\sigma}(x+\mathrm{i} y)=\frac{1}{x-\bar{\mu}+\sigma \bar{h}+\mathrm{i} \operatorname{sgn}(y)(\Delta+|y|)} .
$$

We introduced an effective (renormalized) chemical potential $\bar{\mu}_{\sigma}=E_{d}+U n / 2$ and magnetic field $\bar{h}=h+U m / 2$. The total charge density $n=n_{\uparrow}+n_{\downarrow}$ and magnetization (magnetic moment) at the impurity site $m=n_{\uparrow}-n_{\downarrow}$ are determined from the fully renormalized one-electron local propagator containing the dynamical self-energy $\Sigma_{\sigma}$. That is, we define

$$
n_{\sigma}=\frac{1}{\beta} \sum_{n} G_{\sigma}\left[\mathrm{i} \omega_{n}-\Sigma_{\sigma}\left(\mathrm{i} \omega_{n}\right)\right],
$$

where $\omega_{n}=(2 n+1) \pi T$ are fermionic Matsubara frequencies in units $k_{\mathrm{B}}=1$. The dynamical self-energy $\Sigma_{\sigma}$ will be determined from a diagrammatic expansion.

We apply the diagrammatic expansion not directly to the self-energy but rather to two-particle vertices. In this way we gain a better control of possible singularities in the Bethe-Salpeter equations for the vertex functions. That is also why we must renormalize the dynamics of two-particle functions. The dynamical self-energy $\Sigma_{\sigma}$ (correction to the Hartree term) is then calculated from the two-particle singlet vertex $\Gamma_{\sigma-\sigma}$ via the SchwingerDyson equation

$$
\begin{aligned}
& \Sigma_{\sigma}\left(\mathrm{i} \omega_{n}\right)=-\frac{U}{\beta^{2}} \sum_{m, n^{\prime}} G_{\sigma}\left(\mathrm{i} \omega_{n^{\prime}}\right) G_{-\sigma}\left(\mathrm{i} \omega_{n+m}\right) \\
& \quad \times G_{-\sigma}\left(\mathrm{i} \omega_{n^{\prime}+m}\right) \Gamma_{\sigma-\sigma}\left(\mathrm{i} \omega_{n^{\prime}}, \mathrm{i} \omega_{n} ; \mathrm{i} \nu_{m}\right),
\end{aligned}
$$

where we denoted $\nu_{m}=2 \pi m T$ bosonic Matsubara frequencies.

An external magnetic field leads to spin polarization and split of spin singlet and triplet contributions to the two-particle vertex. To control both these contributions one has to formulate the diagrammatic expansion in a spin-invariant form. It means that one has to treat the singlet and triplet multiple scatterings separately. This can be achieved when we represent the bare interaction as a matrix in spin indices. Assigning the indices so that we assume horizontal propagation of particles from left to right and holes from right to left, whereby the upper indices belong to particles and the lower to holes, we write

$$
U_{\tau \tau^{\prime}}^{\sigma \sigma^{\prime}} \equiv U\left(\delta_{\sigma, \sigma^{\prime}} \delta_{\tau, \tau^{\prime}} \delta_{\sigma,-\tau}-\delta_{\sigma, \tau} \delta_{\sigma^{\prime}, \tau^{\prime}} \delta_{\sigma,-\sigma^{\prime}}\right) .
$$

This matrix is graphically represented in Fig. 1 and makes singlet and triplet electron-hole scatterings equivalent. It casts their contribution into a single channel and a single Berthe-Salpeter equation.

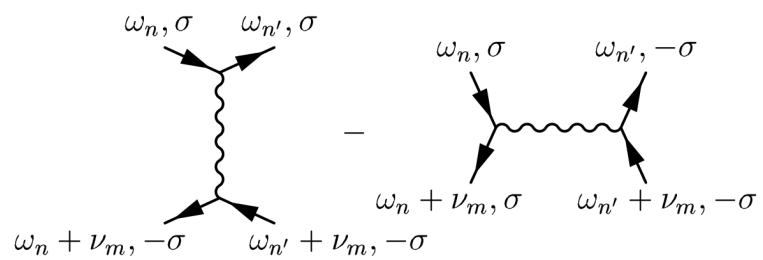

Fig. 1. Graphical representation of the interaction matrix in SIAM with dynamical variables of the interacting fermions.

We now appropriately convert the two-particle vertices into the spin-matrix representation, that is, we replace the vertex $\Gamma_{\sigma \sigma^{\prime}}$ with a matrix $\Gamma_{\tau \tau^{\prime}}^{\sigma \sigma^{\prime}}$. For the bare interaction from Eq. (5) only a few matrix elements are nonzero, since we have to comply with charge and spin conservation. In the charge symmetric (half-filled) case with $\bar{\mu}=0$ we generally have only three independent scalar vertex functions

$$
\begin{gathered}
\Gamma_{\tau \tau^{\prime}}^{\sigma \sigma^{\prime}}=\delta_{\sigma, \sigma^{\prime}} \delta_{\tau, \tau^{\prime}}\left(\Gamma_{\mathrm{t}} \delta_{\sigma, \tau}+\Gamma_{\mathrm{s}} \delta_{\sigma,-\tau}\right) \\
-\delta_{\sigma, \tau} \delta_{\sigma^{\prime}, \tau^{\prime}} \delta_{\sigma,-\sigma^{\prime}} \Gamma_{\overline{\mathrm{t}}} .
\end{gathered}
$$

The subscripts s, t, $\overline{\mathrm{t}}$ refer to singlet, triplet with spin projection $S^{z}=1$ and triplet with $S^{z}=0$, respectively. We determine these three vertices by means of the many-body diagrammatic expansion.

\section{Vertex functions in the Kondo regime}

The principal objective of the diagrammatic expansion of our approach is to determine a reliable expression for the two-particle vertex $\Gamma$ by using the Bethe-Salpeter equations. In particular, we concentrate on critical regions of these equations. Singularities in the BetheSalpeter equations can occur in models with a repulsive interaction only in the electron-hole channel. Critical behavior of vertex functions in impurity models corresponds to the Kondo regime. A fundamental assumption of our construction is that the critical region of these singularities can be reached within a simple approximation, playing the role of a mean-field. Such an approximation is RPA with multiple scatterings, both singlet and triplet ones, in the spin-invariant formulation.

\subsection{RPA vertices}

Before we start with the genuine perturbation expansion, we have to reach a critical region of a singularity 
in the Bethe-Salpeter equation with electron-hole multiple scatterings. The only restriction is that we must not cross the critical value of the interaction strength in the Fermi-liquid regime of impurity models. Criticality in SIAM is reached within RPA with a finite value of the critical interaction strength. This critical interaction sets a natural bound on the applicability of the non-renormalized weak-coupling expansion.

All the RPA electron-hole vertices can be obtained from a single matrix representation as introduced in the preceding section. Alternatively, one can separately sum multiple diagrams for the vertices $\Gamma_{\mathrm{s}}, \Gamma_{\overline{\mathrm{t}}}$ and $\Gamma_{\mathrm{t}}$. Since the irreducible vertex in RPA is the bare interaction, the Bethe-Salpeter equation for the RPA vertices diagonalize and the two-particle propagation reduces to electron-hole spin polarized bubbles $\chi_{\sigma \tau}\left(\mathrm{i} \nu_{m}\right)=$ $\beta^{-1} \sum_{n} G_{\sigma}\left(\mathrm{i} \omega_{n}\right) G_{\tau}\left(\mathrm{i} \omega_{n}+\mathrm{i} \nu_{m}\right)$. The desired vertices then are

$$
\begin{aligned}
\Gamma_{\mathrm{s}}^{\mathrm{RPA}}\left(\mathrm{i} \nu_{m}\right) & =\frac{U}{1+U \chi_{\uparrow \downarrow}\left(\mathrm{i} \nu_{m}\right)}, \\
\Gamma_{\overline{\mathrm{t}}}^{\mathrm{RPA}}\left(\mathrm{i} \nu_{m}\right) & =\frac{U}{1-U^{2} \chi_{\uparrow \uparrow}\left(\mathrm{i} \nu_{m}\right) \chi_{\downarrow \downarrow}\left(\mathrm{i} \nu_{m}\right)}, \\
\Gamma_{\mathrm{t}}^{\mathrm{RPA}}\left(\mathrm{i} \nu_{m}\right) & =\frac{-U^{2} \chi_{\uparrow \downarrow}\left(\mathrm{i} \nu_{m}\right)}{1-U^{2} \chi_{\uparrow \uparrow}\left(\mathrm{i} \nu_{m}\right) \chi_{\downarrow \downarrow}\left(\mathrm{i} \nu_{m}\right)} .
\end{aligned}
$$

Static values of both the singlet $\left(\chi_{\mathrm{s}}=\chi_{\uparrow \downarrow}(0)\right)$ and triplet $\left(\chi_{\mathrm{t}}=\chi_{\uparrow \uparrow}(0)\right)$ bubbles are negative and hence we have critical interaction strengths $U_{\mathrm{s}}=-1 / \chi_{\mathrm{s}}$ and $U_{\mathrm{t}}=-1 / \chi_{\mathrm{t}}$ indicating a pole in the vertex function $\Gamma^{\mathrm{RPA}}$. This pole is spurious and we cannot continue the diagrammatic expansion to and beyond this point. Before we do this, a vertex renormalization must be involved. We can nevertheless use the RPA result in that we fix the position in the critical region below the critical point of the two-particle vertex and renormalize the diagrammatic expansion so that we never leave the critical region of the RPA pole. Such a procedure is consistent unless we meet other singularities not seen in the mean-field RPA solution. There is no evidence for other criticality than that from RPA in SIAM.

\subsection{Kondo scales and rearrangement of perturbation expansion}

We must rearrange the perturbation expansion so that one can remain in the critical region of the RPA pole during the process of summation of diagrams. First thing to do is to define a new small expansion parameter controlling the expansion. To this purpose we introduce a new scale that we call Kondo, since it is related to the Kondo temperature from the strong-coupling regime of the impurity model. The Kondo scale is defined by the smallest value of the denominator in the RPA electron-hole vertices. There are two different denominators, hence we define

$$
a_{\mathrm{s}}=1+U \chi_{\mathrm{s}} \rightarrow 0, \quad a_{\mathrm{t}}=1+U \chi_{\mathrm{t}} \rightarrow 0
$$

as the singlet and the triplet Kondo scale, respectively. Although they are not equal in the spin-polarized case, we assume that they both are much smaller than all other physical scales in the problem. We use the former scale to control the singlet and the latter to control the triplet contributions to physical quantities.

Having identified the Kondo scale we can rearrange the perturbation expansion beyond RPA. We fix the smallest Kondo scale during the summation process and we calculate the corresponding bare interaction strength so that Eq. (10) is obeyed. It means that by adding new diagrammatic contributions we do not change the Kondo scale but rather the bare interaction. Fixing the Kondo scale stabilizes significantly numerical computational schemes for critical quantities. Moreover, the Kondo scale introduces a new controlling small parameter for a classification of diagrammatic contributions beyond RPA. Since the Kondo scale is very small we can expand the denominator of the two-particle vertex in powers of the transfer frequency. The critical point exists only at very low temperatures and it is sufficient to expand the denominator only to linear power of the frequency so that to keep control over the dominant contribution from the two-particle vertex. We then have

$$
\begin{aligned}
1 & +U \chi\left(\mathrm{i} \nu_{m}\right) \rightarrow 1+U \chi+\mathrm{i} U \chi^{\prime} \nu_{m}+\mathrm{O}\left(\nu_{m}^{2}\right) \\
& \doteq a+\mathrm{i} U \chi^{\prime} \nu_{m},
\end{aligned}
$$

where we denoted $\chi^{\prime}=\mathrm{d} \chi(z) /\left.\mathrm{d} z\right|_{z=0}$. We replace the denominator in the RPA vertex by the right-hand side of Eq. (11). The RPA vertices replace the bare interaction in the diagrammatic expansion beyond RPA. They appear in integrals, but only in combinations with regular functions, two-particle propagators. At low temperatures integrals over the RPA vertices lead to large numbers of order $\ln a$. This is the largest scale for intermediate interaction strengths and hence we can use $|\ln a|^{-1}$ as a small parameter controlling the expansion beyond RPA.

\subsection{Effective interactions and two-particle self-consistency}

Fixing the Kondo scale instead of the bare interaction has a very important consequence. The universal critical behavior depending only on the Kondo scale and its logarithm is determined already by RPA. We can view upon RPA as a mean-field description of the Kondo regime. Expansion around RPA does not change the universal features of its critical behavior, since no new singularities emerge beyond RPA in SIAM.

This conclusion apparently does not hold for non-universal critical properties. Such a typical nonuniversal feature is the dependence of the Kondo scale on the bare parameters, interaction strength or external magnetic field. The bare parameters depend on the approximate scheme with the fixed Kondo scale from Eq. (10). To reach the order of magnitude of this dependence we replace the explicit summation of diagrams by an effective construction using a twoparticle self-consistency. We already demonstrated earlier, Refs. $[7,8]$, that a suitable vertex renormalization can be achieved within the parquet approach with a self- 
consistently balanced electron-hole and electron-electron multiple scatterings. We use the fact that we are interested in the critical region of the electron-hole vertex and simplify the structure of the irreducible two-particle vertices used in the parquet theory. We replace the bounded vertices by constants, effective interactions, while keep dynamical only the vertices displaying critical behavior as disclosed in RPA. We know already from the RPA analysis that the irreducible vertices in the electron-hole scattering channel are regular and only the irreducible vertices in the electron-electron channel share the singularity with the RPA vertex, including its dependence on the single controlling frequency. We denote the dynamical irreducible vertices in the electron-electron channel A. In the theory with effective interactions replacing the irreducible vertices from the electron-hole channel we obtain the dynamical vertex by a simple substitution in the RPA vertex $\Lambda(z)=\Gamma^{\mathrm{RPA}}[U \rightarrow \bar{U}](z)$. In the spin-invariant theory we have three effective interactions $\bar{U}_{\mathrm{s}}, \bar{U}_{\overline{\mathrm{t}}}$ and $\bar{U}_{\mathrm{t}}$, whereby the latter is irrelevant for the one-electron spectral function and we neglect it.

The exact parquet equations do not give a prescription how to determine the effective interactions $\bar{U}_{\mathrm{s}}$ and $\bar{U}_{\overline{\mathrm{t}}}$, since they are not fully compatible with a suppression of finite fluctuations of the vertex functions (negligible with respect to $|\ln a|)$. To achieve consistency we have to average the parquet equations to make them compatible with the ansatz of effective interactions. To this purpose we introduce new fermionic dynamical functions

$$
\begin{aligned}
& L_{\mathrm{s}, \overline{\mathrm{t}}}\left(\mathrm{i} \omega_{n}\right)=\beta^{-1} \sum_{n^{\prime}} \Lambda_{\mathrm{s}, \overline{\mathrm{t}}}\left(-\mathrm{i} \omega_{n}-\mathrm{i} \omega_{n^{\prime}}\right) G_{\uparrow}\left(\mathrm{i} \omega_{n}^{\prime}\right) \\
& \quad \times G_{\downarrow}\left(-\mathrm{i} \omega_{n}^{\prime}\right) .
\end{aligned}
$$

After a few simple manipulations, see Ref. [8], we end up in the Kondo regime and at zero temperature with equations defining the effective interactions

$$
\bar{U}_{\mathrm{s}} \psi_{\mathrm{s}}=U \psi_{\mathrm{s}}-L_{\mathrm{s}}, \quad \bar{U}_{\overline{\mathrm{t}}} \psi_{\mathrm{t}}=U \psi_{\mathrm{t}}-L_{\overline{\mathrm{t}}},
$$

where $\psi_{\sigma \tau}=\beta^{-1} \sum_{n} G_{\sigma}\left(\mathrm{i} \omega_{n}\right) G_{\tau}\left(-\mathrm{i} \omega_{n}\right)$ was used to denote the static two-electron bubble and $L=L(0)$.

Solving these equations asymptotically in the Kondo regime, i.e., with the denominators of vertices $\Lambda_{\mathrm{s}, \overline{\mathrm{t}}}$ replaced by the low-frequency expansion from Eq. (11) with $a \rightarrow 0$ we obtain an explicit asymptotic representation of the singlet and triplet Kondo scales in terms of the bare interaction strength

$$
\begin{aligned}
& a_{\mathrm{s}}=\exp \left(-\frac{\pi \psi_{\mathrm{s}}\left|\chi_{\mathrm{s}}^{\prime}\right|^{2}\left(U\left|\chi_{\mathrm{s}}\right|-1\right)}{\left|\chi_{\mathrm{s}}\right| \Im\left[G_{\uparrow}\left(\mathrm{i} 0^{+}\right)^{2} \chi_{\mathrm{s}}^{\prime}\right]}\right), \\
& a_{\mathrm{t}}=\exp \left(-\frac{\pi \psi_{\mathrm{t}}\left|\Im \chi_{\mathrm{t}}^{\prime}\right|\left(U\left|\chi_{\mathrm{t}}\right|-1\right)}{\left|\chi_{\mathrm{t}}\right|\left|G_{\uparrow}\left(\mathrm{i} 0^{+}\right)\right|^{2}}\right) .
\end{aligned}
$$

Both expressions coincide at zero magnetic field and reproduce the result of Ref. [8] that differs from the exact result only by a numerical factor independent of the model parameters. Let us notice that the static value of the derivative of the singlet bubble $\chi_{\mathrm{s}}^{\prime}$ consists in a finite magnetic field from a real and imaginary part unlike the triplet bubble, which is purely imaginary. The one-electron propagators in the above expressions are the
Hartree ones renormalized only on the static level via the total charge and spin densities. We note that expressions in Eq. (14) are meaningful only if the exponents are large negative so that $a_{\mathrm{s}, \mathrm{t}} \rightarrow 0$. Figure 2 shows the dependence of the singlet and triplet Kondo scales on the applied magnetic field. The singlet scale dominates and determines the smallest scale in the strong-coupling regime of a spin-polarized solution.

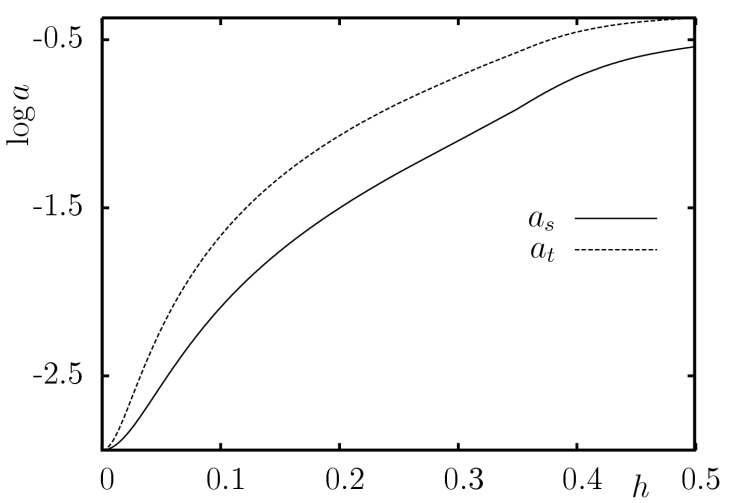

Fig. 2. Dependence of singlet and triplet Kondo scales on the external magnetic field calculated from Eq. (10) with the bare interaction $U=3.138$ and the energy scale $\Delta=1$.

First important conclusion is that relations in Eq. (14) hold for arbitrary filling and magnetic field. The expressions are, however, not universal and depend on the model structure parameter. We must also realize that the exponents are not purely linear in the bare interaction, since the one-electron propagator $G_{\sigma}$ and the two-particle bubbles $\chi$ and $\psi$ depend on the bare interaction via the renormalized parameters $\bar{\mu}$ and $\bar{h}$. Nevertheless, we can see that the critical interaction of the RPA vertex is also a limit defining the strong-coupling regime, that is if $U>1 /|\chi|$. While the genuine Kondo scale sets in only if $U \gg 1 /|\chi|$. The Kondo critical regime exists, as we assumed, for any value of $\bar{\mu}$ and $\bar{h}$, but since the relation between the renormalized chemical potential and magnetic field and the actual charge and spin densities is nonlinear, we cannot easily decide whether the Kondo regime is present at any filling and local magnetization.

\section{Spectral function in the Kondo regime}

The Kondo scale is a two-particle quantity defined here as a static value of the inverse local two-particle vertex $\Gamma$. Experimentally, the Kondo scale is introduced via a Kondo temperature deduced from the saturated value of the inverse static local susceptibility. It is related, but not identical, with the two-particle vertex used in theoretical calculations. Existence of the Kondo behavior is most often demonstrated on the spectral function via an exponentially narrow resonant quasiparticle peak near the Fermi energy. To obtain a self-energy consistent with the Kondo behavior of the two-particle vertex we 
use the Schwinger-Dyson equation, Eq. (4), relating the two-particle vertex and the self energy. We employ the parquet equation in the matrix representation introduced above

$$
\widehat{\Gamma}=\widehat{\Lambda}+\widehat{\bar{U}}-\widehat{U}
$$

representing the full two-particle vertex via the irreducible vertices. We resort to the parquet theory simplified in the Kondo critical regime with dominant critical fluctuations in the electron-electron irreducible vertex. Inserting the explicit solution for the dynamical vertex $\Lambda$ we obtain from Eq. (4) an explicit analytic representation

$$
\begin{aligned}
& \Sigma_{\sigma}\left(\mathrm{i} \omega_{n}\right)=-\frac{U}{\beta} \sum_{m} G_{-\sigma}\left(\mathrm{i} \omega_{n}+\mathrm{i} \nu_{m}\right) \\
& \times\left[\frac{\bar{U}_{\sigma \mathrm{s}}}{1+\bar{U}_{\sigma \mathrm{s}} \chi_{\sigma \mathrm{s}}\left(\mathrm{i} \nu_{m}\right)}-U\right] \chi_{\sigma \mathrm{s}}\left(\mathrm{i} \nu_{m}\right) \\
& -\frac{U}{\beta} \sum_{m} G_{\sigma}\left(\mathrm{i} \omega_{n}+\mathrm{i} \nu_{m}\right) \\
& \times \frac{\bar{U}_{-\sigma \overline{\mathrm{t}}} \chi_{-\sigma \mathrm{t}}\left(\mathrm{i} \nu_{m}\right)}{1-\bar{U}_{\overline{\mathrm{t}}} \bar{U}_{-\overline{\mathrm{t}}} \chi_{\mathrm{t}}\left(\mathrm{i} \nu_{m}\right) \chi_{-\mathrm{t}}\left(\mathrm{i} \nu_{m}\right)} .
\end{aligned}
$$

It can easily be analytically continued to real frequencies and used to obtain the spectral function. We plot the spectral function for both majority and total spins for several values of the applied magnetic field in Fig. 3. We can see that the Kondo peak for the majority spins moves first above the Fermi energy. But for stronger fields the shift to the right is stopped and the quasiparticle peak starts to move backward and crosses the Fermi energy until it merges with the lower satellite Hubbard band. The bare interaction is fixed when the magnetic field is increased. The width of the Kondo resonance peak is getting larger and reaches macroscopic scales soon, cf. Fig. 2. It means that the system leaves the critical region, where the inverse Kondo scale dominates, before the quasiparticle peak merges with the satellite one. Let us notice that the system remains in the critical region of the two-particle vertex only if we fix the renormalized chemical potential $\bar{\mu}$ and magnetic field $\bar{h}$ together with the Kondo scale $a_{\mathrm{s}}$.

The quasiparticle peak shifts only on the scale comparable with the singlet Kondo one, $a_{\mathrm{s}}$. Having an analytic expression for the self-energy we can derive an equation for the center of the quasiparticle peak that we denote $\omega_{\sigma} \propto a_{\mathrm{s}}$. From maximizing the spin-dependent spectral function near the Fermi energy in the Kondo regime we obtain a universal equation

$$
\frac{\Im \Sigma_{\sigma}^{\prime}}{\Re \Sigma_{\sigma}^{\prime}}=-\Im \chi_{\mathrm{s}}^{\prime} \frac{\omega_{\sigma}}{a_{\mathrm{s}}}=\frac{2\left[\sigma \bar{h}-\Re \Sigma_{\sigma}\left(\omega_{\sigma}\right)\right] \Delta}{\left[\sigma \bar{h}-\Re \Sigma_{\sigma}\left(\omega_{\sigma}\right)\right]^{2}-\Delta^{2}},
$$

where we abbreviated $\Sigma^{\prime}=\mathrm{d} \Sigma(\omega) /\left.\mathrm{d} \omega\right|_{\omega=0}$. Since $\Re \Sigma(\omega)$ in the Kondo regime is a fast varying function near the Fermi energy, the sign of the shift depends on the bare interaction strength $U$ via the renormalized magnetic field $\bar{h}$ and also the degree of renormalization contained in the self-energy even in the asymptotic limit $h \rightarrow 0$. The RPA bare interaction leads in weak fields to $\omega_{\sigma} \propto \sigma h$, see

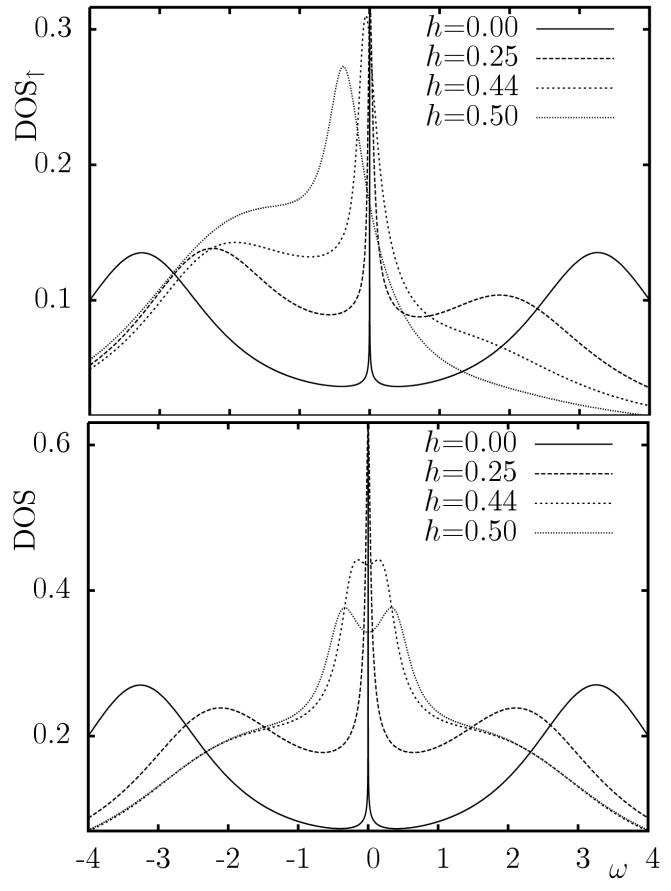

Fig. 3. Spectral density of majority spins with a shifted Kondo resonant peak (upper part) and the total density of states with the split central peak (lower part). The energy scale is $\Delta=1$ and the Kondo scale at zero field is $a=0.00114$ corresponding to the RPA bare interaction $U=3.138$ fixed during the calculations.

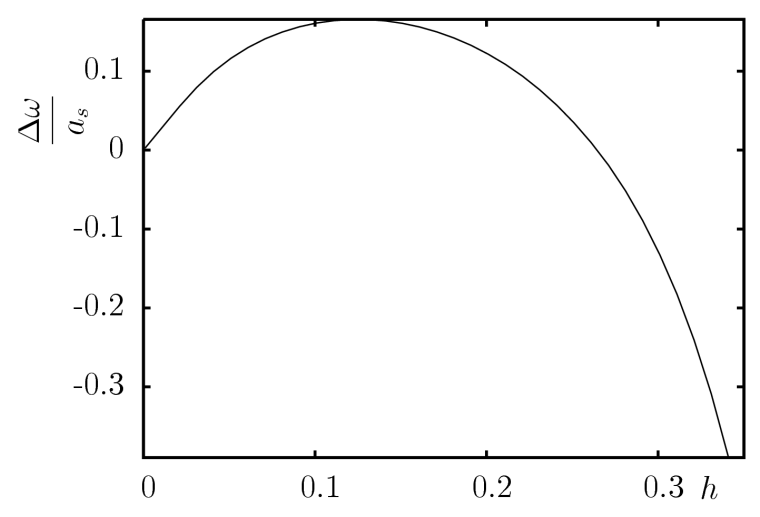

Fig. 4. Shift of the center of the quasiparticle peak for the majority spins as a function of the applied magnetic field evaluated with the RPA values of the input parameters.

Fig. 4. For stronger fields the shift changes the sign. The result for weak magnetic fields seems to be in disagreement with numerical studies of the model [4] and approximate analytic calculations $[5,6]$. The initial direction of the shift obtained by us may, however, be changed in the full parquet self-consistency. Nevertheless, formula (17) is universal and only the explicit form of the lowfrequency asymptotics of the self-energy is approximation dependent. 


\section{Conclusions}

We presented in this paper an analytic method with which one can rather easily trace down the Kondo behavior in impurity models. We showed that the Kondo scale can be introduced already within the random-phase approximation for the electron-hole vertex. Even more, the dependence of the spectral function on the Kondo scale can qualitatively be estimated already within RPA. Advanced approximations with a two-particle self-consistency are needed for non-universal quantities and the dependence of the spectral function in the Kondo regime on the bare input parameters. We proposed a suitable rearrangement of the perturbation expansion beyond RPA in that we fix the (singlet) Kondo scale, $a_{\mathrm{s}}$ together with the renormalized chemical potential $\bar{\mu}$ and the renormalized magnetic field $\bar{h}$. The values of the bare parameters $U, \mu$, and $h$ then depend on the approximation chosen. The small parameter for such a modified expansion is the inverse logarithm of the Kondo scale $\left|\ln a_{\mathrm{s}}\right|^{-1}$. One-parameter scaling guarantees that we can fix the chosen point in the critical region of the pole of the RPA vertex.

We explicitly applied the theoretical construction to study the effects of the external magnetic field on the Kondo behavior of SIAM. We found that the Kondo scale splits in the external magnetic field into singlet and triplet ones related to transversal and longitudinal spin fluctuations, respectively. The former scale was shown to dominate. Further on, we showed that the magnetic field at the fixed bare interaction tends to destroy the Kondo behavior. Weak fields lead to a shift of the central quasiparticle peak and the center of this peak shifts for the majority spins first above the Fermi energy. But for larger fields the peak reverses its upward movement and starts to fall below the Fermi energy where it eventually merges with the lower Hubbard band.

The presented approximation is universal and fully analytically controllable. It can be used as an impurity solver within the dynamical mean-field theory and in the strong-coupling regime of other models with a local electron interaction.

\section{Acknowledgments}

This research was carried out within project AVOZ10100520 of the Academy of Sciences of the Czech Republic and supported in part by grant No. 202/07/0644 of the Grant Agency of the Czech Republic.

\section{References}

[1] A.M. Tsvelick, P.B. Wiegmann, Adv. Phys. 32, 453 (1983).

[2] Y. Meir, N.S. Wingreen, P.A. Lee, Phys. Rev. Lett. 70, 2601 (1993).

[3] J.E. Moore, X.G. Wen, Phys. Rev. Lett. 85, 1722 (2000).

[4] W. Hofstetter, Phys. Rev. Lett. 85, 1508 (2000).

[5] D.E. Logan, N.L. Dickens, J. Phys., Condens. Matter 13, 9713 (2001).

[6] A.C. Hewson, J. Bauer, W. Koller, Phys. Rev. B 73, 045117 (2006).

[7] V. Janiš, P. Augustinský, Phys. Rev. B 75, 165108 (2007).

[8] V. Janiš, P. Augustinský, Phys. Rev. B 77, 085106 (2008). 